\title{
Deciphering the role of a membrane-targeting domain in assisting endosomal and autophagic membrane localization of a RavZ protein catalytic domain
}

\author{
Jui-Hee Park ${ }^{1, \#, ~ S e u n g-H w a n ~ L e e ~}{ }^{1, \#}$, Sang-Won Park ${ }^{1}$, Yong-Woo Jun ${ }^{1}$, Kunhyung Kim ${ }^{2}$, Pureum Jeon ${ }^{3}$, Myungjin Kim $^{2}$, \\ Jin-A Lee ${ }^{3, *} \mathcal{E}$ Deok-Jin Jang ${ }^{1, *}$ \\ ${ }^{1}$ Department of Ecological Science, College of Ecology and Environment, Kyungpook National University, Sangju 37224, ${ }^{2}$ Laboratory of \\ Neurobiochemistry, Emotion, Cognition \& Behavior Research Group, Korea Brain Research Institute, Daegu 41062 , ${ }^{3}$ Department of \\ Biological Science and Biotechnology, College of Life Science and Nano Technology, Hannam University, Daejeon 34054, Korea
}

The bacterial effector protein RavZ from a pathogen can impair autophagy in the host by delipidating the mammalian autophagy-related gene 8 (mATG8)-phosphatidylethanolamine (PE) on autophagic membranes. In RavZ, the membrane-targeting (MT) domain is an essential function. However, the molecular mechanism of this domain in regulating the intracellular localization of RavZ in cells is unclear. In this study, we found that the fusion of the green fluorescent protein (GFP) to the MT domain of RavZ (GFP-MT) resulted in localization primarily to the cytosol and nucleus, whereas the GFP-fused duplicated-MT domain (GFP-2xMT) localized to Rab5- or Rab7-positive endosomes. Similarly, GFP fusion to the catalytic domain (CA) of RavZ (GFP-CA) resulted in localization primarily to the cytosol and nucleus, even in autophagy-induced cells. However, by adding the MT domain to GFP-CA (GFP-CA-MT), the cooperation of MT and CA led to localization on the Rab5-positive endosomal membranes in a wortmannin-sensitive manner under nutrient-rich conditions, and to autophagic membranes in autophagy-induced cells. In autophagic membranes, GFP-CA-MT delipidated overexpressed or endogenous mATG8-PE. Furthermore, GFP-CA $A_{\triangle \alpha 3}-\mathrm{MT}$, an $\alpha 3$ helix deletion within the CA domain, failed to localize to the endosomal or autophagic membranes and could not delipidate overexpressed mATG8-PE. Thus, the CA or MT domain alone is insufficient for stable membrane localization in cells, but the cooperation of MT and CA leads to localization to the endosomal and autophagic mem-

*Corresponding authors. Deok-Jin Jang, Tel: +82-54-530-1213; Fax: +82-54-530-1218; E-mail: jangdj@knu.ac.kr; Jin-A Lee, Tel: +82-42629-8785; Fax: +82-42-629-8789; E-mail: leeja@hnu.kr

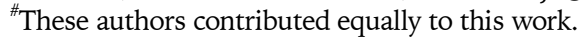

https://doi.org/10.5483/BMBRep.2021.54.2.190

Received 7 September 2020, Revised 30 September 2020, Accepted 26 November 2020

Keywords: Autophagy, Delipidation, mATG8, Membrane-targeting domain, RavZ branes. In autophagic membranes, the CA domain can delipidate $\mathrm{mATG8-PE}$ without requiring substrate recognition mediated by LC3-interacting region (LIR) motifs. [BMB Reports 2021; 54(2): 118-123]

\section{INTRODUCTION}

Autophagy is an evolutionarily conserved cellular degradation pathway that selectively or non-selectively eliminates unwanted materials, such as damaged organelles and harmful cytosolic aggregates, from a cell. Thus, autophagy protects the cell's ability to regulate cellular homeostasis, adapt to various stresses, renovate during development, and prevent genomic damage $(1,2)$. Autophagy was originally believed to be a non-selective degradation pathway in the lysosome. However, accumulated evidence suggests that autophagy may be a selective process guided by specific autophagic receptors and adaptor proteins, which can be classified into different categories, including xenophagy, mitophagy, pexophagy, or reticulophagy (3).

In this study, we turn our focus to xenophagy, which is a selective autophagic process responsible for removing intracellular pathogens, such as cytoplasmic bacteria, viruses, and fungi $(4,5)$. Interestingly, many bacteria have developed mechanisms to inhibit host autophagy. For example, Legionella pneumophila can impair host autophagy through the bacterial effector protein, RavZ. This protein is secreted by Legionella and interferes with autophagy formation by uncoupling the mammalian autophagyregulated gene 8 (mATG8) from phosphatidylethanolamine (PE) on autophagic membranes (6). The RavZ protein delipidates mATG8-PE by hydrolyzing the amide bond between the carboxyl-terminal glycine residue and an adjacent/aromatic residue in mATG8 $(6,7)$. Reportedly, the membrane-targeting (MT) domain and an $\alpha 3$ helix in the catalytic (CA) domain of RavZ are mainly involved in autophagosome targeting (7). Other reports indicate that the LC3-interactging region (LIR) motifs in the RavZ protein contribute to autophagic membrane targeting of the RavZ protein or recognition of mATG8 on the autophagic mem- 
brane $(8,9)$. A recent study demonstrated that the MT domain or LIR motifs of the RavZ protein have complementary functions in RavZ through alternative pathways (10). An RavZ mutant can still delipidate mATG8-PE nonselectively in the absence of three functional LIR motifs, but it can also delipidate mATG8$\mathrm{PE}$ in an LIR motif binding-dependent manner in the absence of an MT domain (10). However, the MT domain function's precise mechanism for intracellular targeting of the RavZ protein in cells has not been elucidated yet.

In this investigation, we found that GFP fused to the RavZ MT domain (GFP-MT) alone was localized to the cytosol and nucleus diffusely, whereas duplication of the MT domain fused to GFP (GFP-2xMT) resulted in localization to early and late endosomes. Similarly, the RavZ CA domain (GFP-CA) alone diffusely localized to the cytosol and nucleus, whereas the combination of MT and CA domains (GFP-CA-MT) led to wortmannin-sensitive endosomal membrane targeting under nutrient-rich conditions and autophagic membrane targeting under autophagic conditions. Similar to wild-type RavZ, on autophagic membranes, GFP-CA-MT could delipidate overexpressed or endogenous LC3B-PE or GABARAP-PE in cells. Collectively, our results suggest that the CA domain has the capacity for delipidating mATG8-PE on autophagic membranes, but alone is insufficient for intracellular membrane localization to endosomal and autophagic membranes. However, the CA domain can be localized to autophagic membrane by the assistance of MT domain and delipidate LC3B-PE or GABARAP-PE nonspecifically and efficiently in cells.

\section{RESULTS AND DISCUSSION}

Endosomal membrane targeting of a duplicated-MT domain We recently demonstrated that an LIR motif-deficient RavZ mutant delipidated overexpressed GFP-fused LC3B-PE or GABARAP-PE efficiently (10), which indicates that the MT domain plays an essential role in RavZ function. However, it still remains unclear how the MT domain compensates for the function of the LIR motif-deficient RavZ. To clarify this, we initially investigated the cellular localization of an MT domain within the RavZ protein. It has been reported that the RavZ MT domain can bind significantly to $\mathrm{PI} 3 \mathrm{P}$-positive liposomes or marginally to $\mathrm{PI}(3,5) \mathrm{P}_{2}-$ positive liposomes in vitro (7). However, as shown in Fig. 1A, GFP fused to the MT domain (GFP-MT) alone resulted in diffuse cytosolic and nuclear localization in established mouse embryonic fibroblast (MEF) cells, which indicates that the MT domain alone is insufficient for intracellular membrane targeting in cells.

Similarly, PI3P binding to the FYVE finger of Hrs or EEA1 is mainly cytosolic when expressed in cells because of its weak binding affinity (11). However, duplication of the FYVE finger of Hrs $(2 \times$ FYVE) results in increased affinity for PI3P and is localized to the endosome membrane (11). Therefore, duplication of the MT domain of RavZ may lead to stable endosome targeting. Therefore, we generated a GFP-fused duplicated-MT

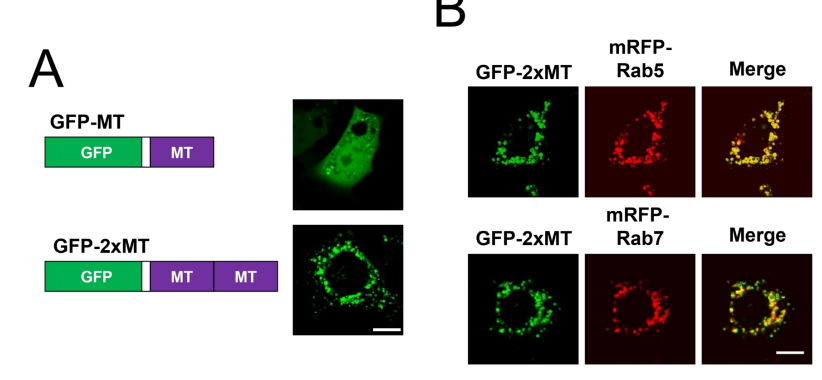

Fig. 1. GFP-2xMT targets early and late endosomes. (A) Schematic diagram of GFP-fused MT (GFP-MT), 2xMT (GFP-2xMT) (left), and confocal images depicting the intracellular localization of GFP-MT or GFP-2xMT in MEF cells (right). Scale bar: $10 \mu \mathrm{m}$. (B) Confocal images showing cellular localization of overexpressed GFP-2xMT with mRFP-Rab5, or -Rab7 in MEF cells. Scale bar: $10 \mu \mathrm{m}$.

domain (GFP-2xMT) and expressed the construct in MEF cells. In contrast to GFP-MT, GFP-2XMT showed clear intracellular vesicle localization within the MEF cells (Fig. 1A). GFP-2xMT was co-localized with mRFP-Rab5, an early endosome marker, and with mRFP-Rab7, a late endosome marker, which indicates clear early/late endosome localization in cells, as shown in Fig. 1B. Taken together, our results suggest that the MT domain alone is insufficient for endosomal membrane targeting. However, duplication of the MT domain can lead to stable intracellular membrane targeting in cells.

\section{Endosomal membrane targeting of the RavZ CA domain aided by the MT domain}

The $\alpha 3$ helix in the CA domain of the RavZ protein is reportedly involved in membrane association (7). However, as shown in Fig. 2A, GFP fused to CA domain (GFP-CA) alone exhibits diffuse cytosolic and nuclear localization. However, if the MT domain is attached to the CA domain, it localizes to an intracellular membrane, such as the endosome. To test this, we generated a GFP-MT fused to the RavZ catalytic domain (GFP-CAMT) and expressed it in MEF cells. Unlike GFP-MT or GFP-CA alone, GFP-CA-MT was clearly localized to the intracellular membrane (Fig. 2A). The relative ratio of GFP fluorescence intensity to the endosomal membrane and cytoplasm ( $\mathrm{M} / \mathrm{C}$ ratio) clearly showed that the membrane localization of GFP-CA-MT was much enhanced compared with that of GFP-MT or GFP-CA alone (Fig. 2B). In contrast, GFP-CA $A_{\triangle \alpha 3}-M T$, a deletion of the $\alpha 3$ helix within the $C A$ domain, exhibited reduced endosome localization (Fig. 2A and 2B). Additionally, GFP-CA-MT was co-localized with Rab5-mRFP under nutrient-rich conditions, indicating early endosome targeting (Fig. 2C). Reduced GFP-CA-MT endosome localization was observed in the presence of wortmannin, a general PI3 kinase inhibitor (Fig. 2D). As a control, GFP-AKT1-PH, a $\mathrm{PI}(3,4,5) \mathrm{P}_{3}$-binding motif, exhibited reduced plasma membrane localization in the presence of wortmannin; however, the plasma membrane and endosome localization of 


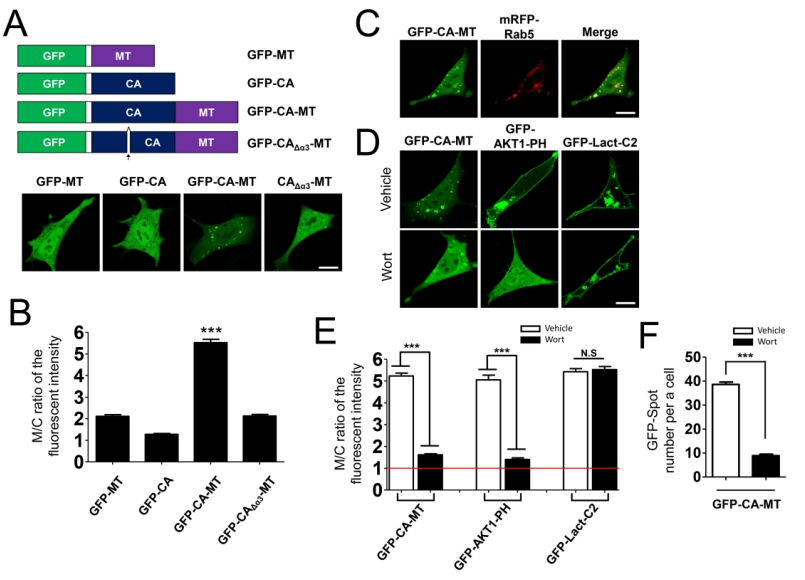

Fig. 2. Endosome localization to GFP-CA-MT in a wortmannin-sensitive manner. (A) Schematic diagram of several GFP-fused MT (GFP-MT), CA (GFP-CA), CA-MT (GFP-CA-MT), or $\mathrm{CA}_{\Delta \alpha 3}-\mathrm{MT}\left(\mathrm{GFP}_{-\mathrm{CA}} \mathrm{A}_{\Delta \alpha 3}-\mathrm{MT}\right) \mathrm{do}$ mains (upper). CA, catalytic domain; MT, membrane-targeting domain. Confocal images (bottom) depicting the intracellular localization of GFP-MT, GFP-CA, GFP-CA-MT, or GFP-CA ${ }_{\Delta \alpha 3}-M T$ in MEF cells. Scale bar: $10 \mu \mathrm{m}$; bar graphs. (B) Quantification data showing the GFP fluorescence intensity of endosomal or plasma membrane and cytosol (the MC ratio) in GFP-MT-, GFP-CA-, GFP-CA-MT-, or GFP-CA $\Delta \alpha 3-$ MT-expressing cells ( $n=25$ for each group). The data are presented as the mean + SEM. $* * * P<0.001$, according to one-way analysis of variance (ANOVA), followed by Tukey's post-hoc test. (C) Early endosome localization of GFP-CA-MT in MEF cells. Scale bar: $10 \mu \mathrm{m}$. (D) Confocal images depicting the targeting of GFP-CA-MT in the absence or presence of a wortmannin-sensitive (Wort, $200 \mathrm{nM}$ in DFP for an hour) manner in MEF cells by using the intracellular localization of GFP-AKT1-PH or GFP-Lact-C2. Scale bar: $10 \mu \mathrm{m}$; bar graphs. (E) Illustration of GFP fluorescence intensity of the endosomal or plasma membrane and cytosol (MC ratio) ( $n=10$ for each group). Values are presented as the mean + SEM. ${ }^{* * * P}<0.001$ (two-tailed unpaired Student's t-test). (F) GFP-positive spots number before and after wortmannin in GFP-CA-MT expressing cells $(n=10$ for each group). Values are presented as the mean + SEM. $* * * \mathrm{P}<0.001$ (two-tailed unpaired Student's t-test).

GFP-Lact-C2, a PS binding motif, did not change in the presence of wortmannin (Fig. 2D). The M/C ratio and the number of GFP-positive spots clearly showed that membrane localization of GFP-CA-MT was greatly reduced in the presence of wortmannin (Fig. 2E and 2F). These results indicate that the presence of the $\alpha 3$ helix in both the CA and MT domains is required for endosome localization of GFP-CA-MT in a wortmannin-sensitive manner under nutrient-rich conditions, suggesting that $\mathrm{PI} 3 \mathrm{P}$ or $\mathrm{PI}(3,5) \mathrm{P}_{2}$-positive membrane association is involved in endosome targeting.

Taken together, our data clearly shows that the CA or MT domains alone failed to localize to endosomes in cells, whereas the cooperation of the MT domain and the $\alpha 3$ helixes within the CA domain and can lead to endosomal membrane localization in a wortmannin-sensitive manner. Therefore, the MT domain assists the CA domain of the RavZ protein in endosome membrane targeting.
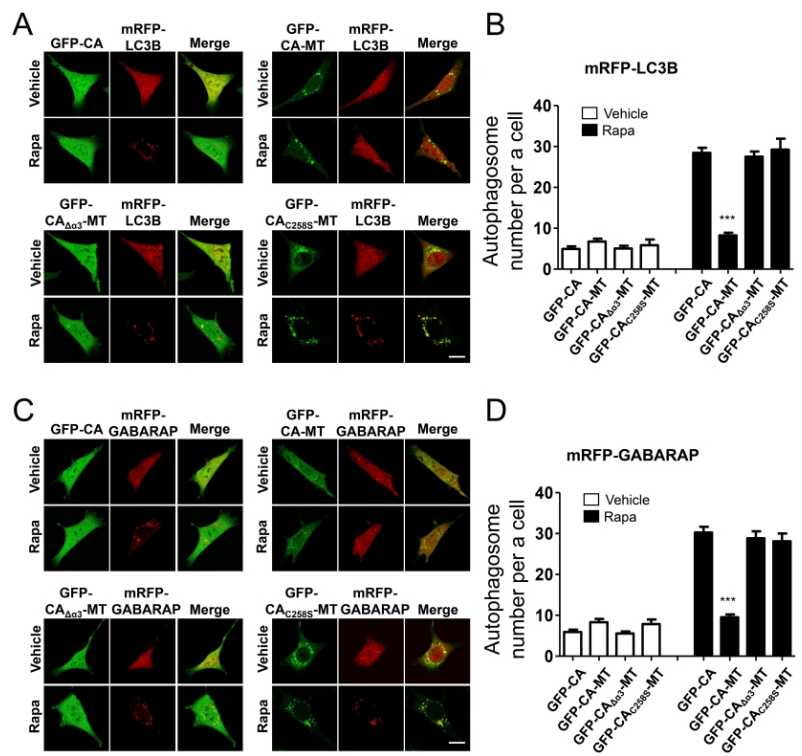

D

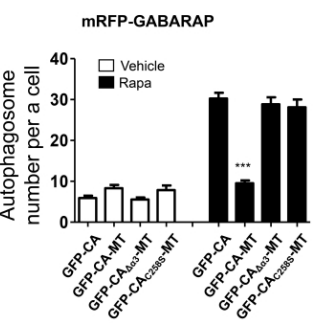

Fig. 3. Effects of GFP-CA-MT overexpression on the delipidation of overexpressed mATG8 protein in rapamycin-induced autophagy. (A, C) Confocal images showing cellular localization and delipidation of mRFP-LC3B (A) or mRFP-GABARAP (C) co-expressed with GFP-CA, GFP-CA-MT, GFP-CA ${ }_{\Delta \alpha 3}-\mathrm{MT}$, or GFP-CA $2585-\mathrm{MT}$ in MEF cells upon autophagy induction (100 nM rapamycin [Rapa] for 2-4 hours). Scale bar: $10 \mu \mathrm{m}$. (B, D) The bar graphs illustrate the autophagosome spot numbers per cell in mRFP-LC3B-expressing cells (B) or mRFP-GABARAPexpressing cells (D) ( $n=25$ for each group). Data are presented as mean + SEM. $* * * P<0.001$ (one-way analysis of variance (ANOVA) followed by Tukey's post-hoc test).

\section{Delipidation of overexpressed mATG8s by GFP-CA-MTs}

The role of LIR motifs in the delipidation process by the RavZ protein remains controversial. It has been proposed that LIR motifs play a key role in RavZ functions, including autophagic membrane localization and substrate recognition $(8,12)$. For example, the LIR2 motif of RavZ contributes to substrate recognition in the autophagic membrane (12). On the other hand, it has been proposed that LIR motifs exhibit marginal roles in RavZ functions. For example, our group recently showed that an LIR motif-deficient RavZ mutant could still delipidate LC3B-PE or GABARAP-PE (10).

To clearly verify this, we determined whether a GFP-CA-MT, which has no LIR motifs, can delipidate overexpressed mATG8-PE in MEF cells. With rapamycin treatment, GFP-CA, GFP-CA-MT,

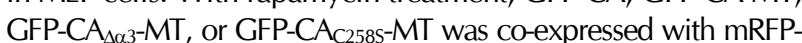
LC3B or mRFP-GABARAP and induced autophagy in MEF cells (Fig. $3 \mathrm{~A}$ and $3 \mathrm{C}$ ). As shown in Fig. 3A-D, overexpression of GFP-CA or GFP-CA ${ }_{\Delta \alpha 3} 3$ MT did not lower mRFP-LC3B-positive or mRFP-GABARAP-positive autophagic membrane formation and was not localized to the autophagic membrane in MEF cells. Furthermore, overexpression of GFP-CA-MT decreased mRFP-LC3B-positive and mRFP-GABARAP-positive autophagic membrane formation in rapamycin-treated MEF cells (Fig. 


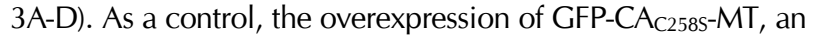
enzyme-deficient mutant, did not lower mRFP-LC3B- or mRFPGABARAP-positive autophagic membrane formation in rapamycintreated MEF cells (Fig. 3A-D). However, GFP-CA $22585-M T$ was localized to the autophagic membrane (Fig. 3A and 3C). Thus, considering the autophagic membrane localization of GFP-CA $\mathrm{C}_{2585} \mathrm{~s}^{-}$ MT, GFP-CA-MT could be localized to the autophagic membrane via the MT domain and the $\alpha 3$ helix of CA domain, and delipidate overexpressed mRFP-LC3B-PE or mRFP-GABARAP-PE on the autophagic membrane nonspecifically.

\section{Delipidation of endogenous mATG8 by GFP-CA-MT}

Next, to further investigate whether GFP-CA-MT can delipidate endogenous mATG8-PE, we used Western blot analysis to measure the endogenous LC3B-II or GABARAP-II levels in GFP-CAMT-expressing HEK293T cells. As shown in Fig. 4A and 4B, overexpression of GFP-CA-MT, but not GFP-CA 2585 -MT, effectively reduced endogenous LC3B-II levels similarly to that of GFP-RavZ in rapamycin-treated cells. Similarly, endogenous GABARAP-II was reduced by the overexpression of GFP-CA-MT or GFP-RavZ, but not GFP or GFP-CA ${ }_{\mathrm{C} 258 \mathrm{~s}} \mathrm{MT}$ in rapamycinplus-CQ-treated cells (Fig. 4C and 4D). Our results clearly demonstrated that GFP-CA-MT can delipidate endogenous LC3B-PE and GABARAP-PE on autophagic membranes as a result of rapamycin-induced autophagy.

To further confirm endogenous mATG8s delipidation, we used endogenous autophagic membrane-detecting probes, which were described previously $(13,14)$. GFP-CA, GFP-CA-MT, or GFP-

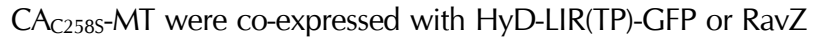
$(\triangle \mathrm{CA})_{\mathrm{Fy}} \mathrm{GFP}$, which can detect all types of endogenous mATG8positive or LC3 subfamily-positive autophagic membrane in cells, respectively $(13,14)$. As shown in Fig. 4E-G, GFP-CA or GFP$\mathrm{CA}_{\mathrm{C} 258 \mathrm{~s}-\mathrm{MT}}$ did not reduce the HyD-LIR(TP)-GFP-positive or $\operatorname{RavZ}(\triangle \mathrm{CA})_{\mathrm{Fy}}$-GFP-positive autophagic membrane compared with GFP expressing cells, whereas GFP-CA-MT almost completely abolished HyD-LIR(TP)-GFP-positive and $\operatorname{RavZ}(\Delta \mathrm{CA})_{\mathrm{F} y^{-}}$ GFP-positive autophagic membrane in rapamycin-treated MEF cells, which is similar to that of GFP-RavZ. These results clearly indicate that GFP-CA-MT is sufficient for delipidating all types of endogenous mATG8-positive autophagic membranes, including those of the LC3 subfamily. As a control, GFP-CA $2585-M T$ did not reduce and was co-localized to an HyD-LIR(TP)-GFP-positive or $\operatorname{RavZ}(\triangle \mathrm{CA})_{\mathrm{Fy}}$-GFP-positive autophagic membrane (Fig. $4 \mathrm{E}-\mathrm{G})$. Taken together, our results clearly show that GFP-CA-MT, which contains no internal LIR motifs, can efficiently localize to the autophagic membrane and delipidate all types of endogenous or overexpressed mATG8s on autophagic membranes as efficiently as wild-type RavZ. These results clearly indicate that the MT domain plays a key role in RavZ function.

In this investigation, we clearly showed that the RavZ CA domain has the capacity to delipidate all types of mATG8-PE in autophagic membranes without requirment of any additional domains, but alone it is insufficient for intracellular membrane localization to endosomal and autophagic membranes. Similarly,
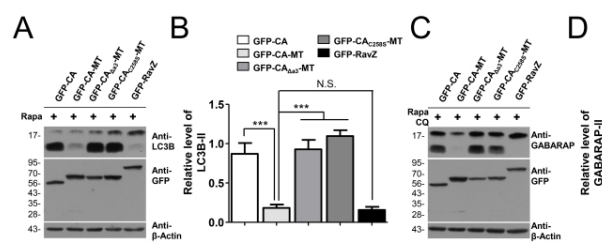

$\mathrm{D}$

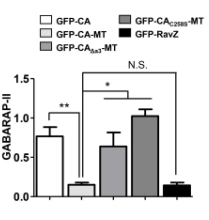

E

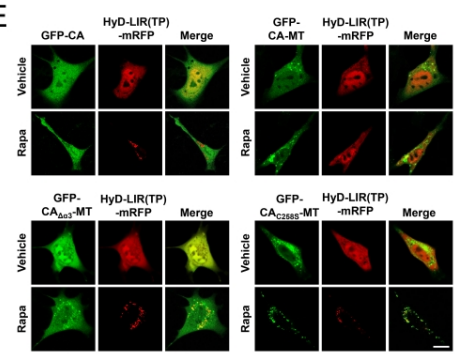

$\mathrm{F}$

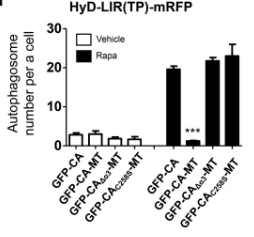

G

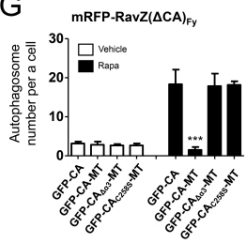

Fig. 4. Effects of the overexpression of GFP-CA-MT on the delipidation of endogenous mATG8 protein in rapamycin-induced autophagy. (A, B) Representative Western blots (A) of four independent experiments of endogenous LC3B in cells expressing various GFP-fused RavZ domain constructs in HEK293T cells upon autophagy induction (100 nM rapamycin [Rapa] for 4 hours) and quantification analysis (B) with an anti-GFP, anti-LC3B, or anti- $\beta$-actin antibody. The data are presented as the mean + SEM of four independent experiments. N.S., not significant. $* * * P<0.001$ (repeated measures one-way analysis of variance (ANOVA) followed by Tukey's post-hoc test) (C, D) Representative western blots $(C)$ of five independent experiments of endogenous GABARAP in cells expressing the GFP-RavZ protein or its mutants in HEK293T cells upon autophagy induction (100 nM rapamycin [Rapa] + $50 \mu \mathrm{M}$ chloroquine (CQ) 4 hours) and quantification analysis (D) with an anti-GFP, anti-GABARAP, or anti- $\beta$-actin antibody. The data are presented as the mean + SEM of five independent experiments. N.S., not significant. ${ }^{*} P<0.05$, $* * P<0.01$ (repeated measures ANOVA) followed by Tukey's post-hoc test) (E, F) Confocal images (E) and quantification (F) of the autophagosome spot number in cells expressing various GFP-fused RavZ domain constructs, co-expressed with HyD-LIR(TP)-mRFP-positive autophagosomes in MEF cells upon autophagy induction (100 nM rapamycin [Rapa] 2-4 hours). Scale bar: $10 \mu \mathrm{m}$; for quantitative analysis of the autophagosome spot number ( $\mathrm{n}=25$ for each group). Data are presented as mean + SEM. ***P $<0.001$ (one-way analysis of variance (ANOVA) followed by Tukey's post-hoc test). (G) Quantification of the autophagosome spot number in cells expressing various GFP-fused RavZ domain constructs, co-expressed with mRFP-RavZ $(\triangle C A)_{\mathrm{Fy}}$ positive autophagosomes in MEF cells upon autophagy induction (100 nM rapamycin [Rapa] 2-4 hours). Scale bar: $10 \mu \mathrm{m}$. For quantitative analysis of the autophagosome spot number ( $n=25$ for each group). Data are presented as mean + SEM. $* * * P<0.001$ (ANOVA followed by Tukey's post-hoc test).

the RavZ MT domain alone is insufficient for intracellular membrane localization to endosomal and autophagic membranes. However, the cooperation of the two domains including the MT domain and the $\alpha 3$ helix of the CA domain leads to endosomal and autophagic membrane targeting. The CA domain deli- 
pidates all types of mATG8-PE nonspecifically and efficiently on the autophagic membrane without any requirement for mATG8 binding mediated by LIR motifs on the autophagic membrane.

\section{MATERIALS AND METHODS}

\section{DNA constructs}

All primer sequences used in these experiments are listed in Supplementary Table 1. MT, 2xMT, CA, CA-MT, and CA(C258S) were generated by PCR amplification of pcDNA3.1(-)-FlagRavZ and pcDNA3.1(-)-Flag-RavZ(C258S) vectors and inserted into the N3-GFP or C1-GFP vectors using each restriction enzyme set. CA( $(\Delta \alpha 3)-M T$ was amplified by overlap extension PCR with C1-CFP-CA-MT used as a template, and then was inserted into the C1-GFP vector using the restriction enzyme set. GFPAKT1-PH, GFP-Lact-C2, mRFP-Rab5 and mRFP-Rab7 were provided by Addgene (Cambridge, MA, USA). We used previously described DNA constructs for mRFP-LC3B, and mRFP-GABA RAP in this study.

\section{Cell culture and transfection}

HEK293T cells and established MEF cells were cultured in Dulbecco's modified Eagle's medium (DMEM) and supplemented with $10 \%(\mathrm{v} / \mathrm{v})$ fetal bovine serum (FBS) and penicillin/streptomycin in a humidified atmosphere with $5 \%(\mathrm{v} / \mathrm{v}) \mathrm{CO}_{2}$ at $37^{\circ} \mathrm{C}$. Cells were seeded in a 6-well cell culture plate (Catalog \#: 30006; SPL, Gyeonggi, Korea) or a sticky-slide eight-well system (Catalog \#: 80828; Ibidi, Martinsried, Germany) to obtain $40 \%-60 \%$ confluent cells on the day of imaging. Cells were transfected with the indicated DNA constructs using calcium phosphate (Catalog \#: 631312; Takara Bio inc., Shiga, Japan) or Lipofectamine 2000 (Life Technologies, Carlsbad, CA, USA) 24-26 $\mathrm{h}$ before analysis. The relative amount of each construct was empirically calculated according to the relative expression of each construct combination.

\section{Confocal microscopy and drug treatment}

Fluorescent images were obtained with an inverted Zeiss LSM-700 scanning laser confocal microscope operated by ZEN software (Carl Zeiss, Oberkochen, Germany). The laser lines for excitation and spectral detection windows for the fluorochromes measured $488 \mathrm{~nm}$ (508-543 nm for GFP) and $561 \mathrm{~nm}$ (578-649 $\mathrm{nm}$ for mRFP). We used appropriate GFP (500-550 nm) and mRFP (575-625 nm) emission filters for sequential imaging of each fluorescent protein. Rapamycin and chloroquine (CQ) were acquired from Sigma-Aldrich (St. Louis, MO, USA). Autophagy was induced by incubating cells with $100 \mathrm{nM}$ rapamycin in the presence or absence of $50 \mu \mathrm{M} \mathrm{CQ}$ in DMEM + 10\% FBS for $4 \mathrm{~h}$. We conducted all treatments and assays at $37^{\circ} \mathrm{C}$, unless otherwise indicated. Wortmannin, a nonspecific covalent inhibitor of phosphoinositide 3-kinase, (Sigma-Aldrich, W1628) was used at a concentration of $200 \mathrm{nM}$ in diisopropylfluorophosphate (DFP) for one hour.

\section{Western blot analysis}

Transfected HEK293T cell lysates were prepared by adding cells to a lysis buffer solution (50 mM Tris, pH 7.5, $150 \mathrm{mM}$ $\mathrm{NaCl}, 0.5 \%$ sodium deoxycholate, $0.2 \%$ SDS, $0.2 \% \mathrm{NP}-40$, and protease inhibitors). Equal amounts of protein were resolved by SDS-PAGE, transferred to polyvinylidene fluoride (PVDF) membranes, and incubated with primary antibodies overnight at $4^{\circ} \mathrm{C}$. After three washes, we incubated membranes with secondary antibodies conjugated with horseradish peroxidase (HRP) for 1 h. Signals were visualized with an Advansta WesternBright ECL kit (K-12045-D50). We used the following antibodies: GABARAP (Cell Signaling, \#13733S) at 1:1,000, LC3 (Cell Signaling, \#2775) at $1: 1000, \beta$-actin (Santa Cruz Biotechnology, sc-47778) at 1:1000, and goat anti-mouse HRP (Santa Cruz Biotechnology, sc-2005) or goat anti-rabbit HRP antibody (Santa Cruz Biotechnology, sc-2313) at 1:10,000. To quantify the intensity of the western blot bands, the area of each band was quantified using the Image program. Band quantitation was obtained from three independent experiments. All statistical data were calculated and graphed using GraphPad Prism5 (GraphPad, Inc., La Jolla, CA, USA).

\section{Spot number analysis}

To find a reduction in mATG8-positive autophagosomes by enzyme activation of RavZ in autophagy-induced cells, we counted the number of spots over a certain field size in a single cell using the ImageJ software. The cell image was changed to an 8-bit image and inverted. The background was then removed so that only the spots remained visible. Finally, we counted the number of spots using the "Analyze Particles" function of ImageJ. A minimum of 20 cells was quantified using this approach. All statistical data were calculated and graphed using GraphPad Prism5 (GraphPad, Inc., La Jolla, CA, USA).

\section{Quantitative analysis of the $\mathrm{M} / \mathrm{C}$ ratio}

To calculate the ratio of membrane to cytosol (M/C) fluorescent intensities, the average value of the vesicle or cytosol fluorescent intensity was obtained from at least five randomly selected points on the vesicles or in the cytosol of a single cell using ZEN software. Similarly, the quantitative MVC ratio of at least 25 randomly selected cells per experiment was obtained from three independent experiments. All statistical data were calculated and graphed using GraphPad Prism5 (GraphPad, Inc., La Jolla, CA, USA).

\section{ACKNOWLEDGEMENTS}

JA Lee was supported by the Basic Research Program of the National Research Foundation (NRF-2020R1A2C2005021), the Neurological Disorder Research Program (NRF-2020M3E5D90 79908), and the Bio \& Medical Technology Development Program of the NRF funded by the Ministry of Science \& ICT (2017M3A9G7073521). DJ Jang was supported by the Basic Research Program of the National Research Foundation (NRF-2020 
R1F1A1069564). MK was supported by Mid-career Researcher Program (NRF-2019R1A2C1011083), KBRI basic research program (20-BR-02-07) funded by the Korea government (MSIT).

\section{CONFLICTS OF INTEREST}

The authors have no conflicting interests.

\section{REFERENCES}

1. Levine B and Kroemer G (2019) Biological functions of autophagy genes: a disease perspective. Cell 176, 11-42

2. Lee YK and Lee JA (2016) Role of the mammalian ATG8/ LC3 family in autophagy: differential and compensatory roles in the spatiotemporal regulation of autophagy. BMB Rep 49, 424-430

3. Gatica D, Lahiri V and Klionsky DJ (2018) Cargo recognition and degradation by selective autophagy. Nat Cell Biol 20, 233-242

4. Sharma V, Verma S, Seranova E, Sarkar, S and Kumar D (2018) Selective autophagy and xenophagy in infection and disease. Front Cell Dev Biol 6, 147

5. Kwon DH and Song HK (2018) A structural view of xenophagy, a battle between host and microbes. Mol Cells 41, 27-34

6. Choy A, Dancourt J, Mugo B et al (2012) The Legionella effector RavZ inhibits host autophagy through irreversible
Atg8 deconjugation. Science 338, 1072-1076

7. Horenkamp FA, Kauffman KJ, Kohler LJ et al (2015) The Legionella anti-autophagy effector RavZ Targets the autophagosome via PI3P- and curvature-sensing motifs. Dev Cell 34, 569-576

8. Kwon DH, Kim S, Jung YO et al (2017) The 1:2 complex between RavZ and LC3 reveals a mechanism for deconjugation of LC3 on the phagophore membrane. Autophagy $13,70-81$

9. Kwon DH, Kim L, Kim BW et al (2017) A novel conformation of the LC3-interacting region motif revealed by the structure of a complex between LC3B and RavZ. Biochem Biophys Res Commun 490, 1093-1099

10. Park SW, Jun YW, Jeon P et al (2019) LIR motifs and the membrane-targeting domain are complementary in the function of RavZ. BMB Rep 52, 700-705

11. Dumas JJ, Merithew E, Sudharshan E et al (2001) Multivalent endosome targeting by homodimeric EEA1. Mol Cell 8, 947-958

12. Yang A and Pantoom S (2017) Elucidation of the anti-autophagy mechanism of the Legionella effector RavZ using semisynthetic LC3 proteins. Elife 6, e23905

13. Park SW, Jeon P, Jun YW et al (2019) Monitoring LC3- or GABARAP-positive autophagic membranes using modified RavZ-based probes. Sci Rep 9, 16593

14. Lee YK, Jun YW, Choi HE et al (2017) Development of LC3/GABARAP sensors containing a LIR and a hydrophobic domain to monitor autophagy. EMBO J 36, 1100-1116 